RESUMEN

\title{
Comunicación política
} y personalización de contenidos: ies Twitter el nuevo medio de referencia para la interacción entre gobernantes y gobernados?

\section{Political Communication and} Personalization of Contents:

Is Twitter the new Reference Media for Interactions between Politicians and Citizens?

\section{Roberto Gelado Marcos}

Universidad San Pablo-CEU (Madrid, España) roberto.geladomarcos@ceu.es Belén Puebla Martínez Universidad Rey Juan Carlos (Madrid, España) belen.puebla@urices Rainer Rubira García Universidad Rey Juan Carlos (Madrid, España) rainer.rubira@uric.es
Gelado Marcos, R., Puebla Martínez, B.y Rubira García, R. (2019)

Comunicación política y personalización de contenidos: ¿es Twitter el nuevo medio de referencia para la interccion entre gobernantes y gobernados? Revista Internacional de Investigación en Comunicación aDResearch ESIC. NN 20 Vol 20

Segundo semestre, julio-diciembre 2019. Págs. 8 a 19 https://doi.org/10.7263/adresic-020-01
Clasificación JEL: D72, D83, L86

Palabras clave:

Twitter,

comunicación polític

opinión pública, interactividad
JEL Classification:

72, D83, L86

Key words:

Twitter,

political communi-

cation,

public opinion, interactivity
Objetivo: El objetivo de esta investigación es arrojar luz sobre cómo la eclosión de las redes sociales ha afectado a la comunicación política en los nuevos soportes. La ruptura de barreras entre emisores y receptores y la reconversín potennit de todos ellos en comunicadores de

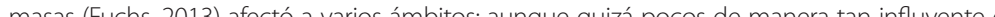
la configuración de la esera socia como la comunicación poltica Ejemplos como la primera

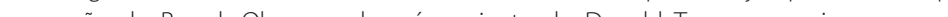
efectivamente, Twitter forma parte integral de las estrategias de comunicación politica.

Diseño/metodología: La investigación se ha articulado mediante un análisis comparativo de la actividad discursiva en Twitter del presidente de los Estados Unidos, Donald Trump, la primera ministra de Reino Unido, Theresa May, y el presidente del gobierno español, Pedro Sánchez, entre los meses de septiembre y diciembre de 2018. Para ello, se ha diseñado una metodología que utiliza el análisis de contenido asistido por ordenador mediante el programa NVivo 11.

Resultados: Los resultados invitan a secundar las dudas expresadas por varios autores recogidos en nuestro estado de la cuestion al respecto del potencial factor de dinamizador de Twitter en relación entre gobernantes y gobernados.

Limitaciones/implicaciones: Pese a la limitación de cuentas analizadas, se puede colegir de este análisis internacional que la contribución de Twitter al enriquecimiento del debate públice es, cuanto menos, dudosa.

Originalidad/contribución: Tanto la visualización de los ejes discursivos de los políticos analizados como el doble enfoque, cuantitativo y cualitativo del estudio de las herramientas temáticas y retónicas que ariculan los discursos de los politicos analizados pueden servir de punto de partida para el anáisis del papel que desempeña Twitter tanto en las estrategias de comunicación política como en su contribución a la esfera pública.

Purpose: The aim of this research is to shed light on how the emergence of social networks has affected political communication in new media. The breaking down of barriers between transmitters and receivers and the potential reconversion of all of them into mass communicators (Fuchs, 2013) affected several spheres; although perhaps only few were affected in such an influential way as the configuration of the social sphere in political communication. Examples such as Barack Obama's first campaign or Donald Trump's more recent campaign lead us to think that Twitter is indeed an integral part of political communication strategies.

Design/methodology: The research has been carried out through a comparative analysis of discursive activity on Twitter of the President of the United States, Donald Trump, the Prime Minister of the United Kingdom, Theresa May, and the President of the Spanish government Pedro Sánchez, between the months of September and December 2018. For this purpose, a methodology has been designed that uses computer-assisted content analysis using the NVivo 11 program.

Findings: The results invite us to confirm doubts expressed by several authors collected and presented in our research regarding the potential dynamizing factor of Twitter in the relationship between the governors and the governed.

Research limitations/implications: Despite the limited number of cases analysed, it can be inferred that the contribution of Twitter to the enrichment of public debate is to say, at the least, dubious.

Originality/value: Both the visualisation of the discourse lines of the analysed politicians and the double focus of the study, quantitative and qualitative, on the thematic tools and rhetoric that charas of the study, quan by the and qual tative, on the thenatic tools and point for the analysis of the role that Twitter plays both in political communication strateng point for the analysis of the role that Twitter plays both in political communication strategies
and in its contribution to the public sphere. 


\section{Introducción}

La eclosión de las redes sociales confirmó una evolución sin retorno de la Web clásica y un rediseño de los modelos de relación entre usuarios. La ruptura de barreras entre emisores y receptores, y la reconversión potencial de todos ellos en comunicadores de masas afectó a varios ámbitos; aunque quizá pocos de manera tan influyente en la configuración de la esfera social como la comunicación política. De pronto, los gobernantes se vieron con herramientas - Twitter, por ejemplo-para interactuar directamente con sus gobernados y estos últimos también se vieron atraídos por la idea de una participación social estimulada. Ejemplos como la primera campaña de Barack Obama o la más reciente de Donald Trump empujan a pensar que, efectivamente, Twitter forma parte integral de las estrategias de comunicación política; pero, ise ha hecho este diálogo efectivo o simplemente se han trasladado viejas formas a nuevos contenedores?

El presente artículo se plantea como objetivos principales arrojar más luz sobre la eficacia real de la interacción entre gobernantes y gobernados en Twitter en diferentes países y estudiar la naturaleza de los temas abordados por los políticos examinados en sus cuentas de esta red social.

\section{Antecedentes teóricos}

Las redes sociales en las nuevas estrategias de comunicación política

La llegada de la Web 2.0 y la apuesta decidida por herramientas que enfatizaran el aspecto interactivo de la Red de Redes, muchísimas disciplinas profesionales experimentaron un cambio irreversible tanto en sus rutinas de producción como en sus objetivos y en sus públicos. La política, en general, y la comunicación política, en particular, han sido, quizá dos de las esferas donde más notable se ha hecho este cambio.
Holmes y McNeal (2016), por ejemplo, hablan de las redes sociales como un elemento con un extraordinario potencial polarizador de la esfera política. Richey (2013) apunta que, del mismo modo que se pueden vislumbrar posibles efectos negativos derivados del influjo de las redes sociales, éstas también pueden ser una herramienta poderosa en la mejora de la competencia cívica. Vatikiotis (2016), por su parte, recuerda el poder de las redes en el desarrollo del activismo político que hemos presenciado durante estos albores del siglo XXI

Semeitko y Scammell (2012, p. 200) ponen todo este impacto en perspectiva afirmando que, sea como fuere, la realidad indiscutible es que las redes sociales se han convertido en el eje central de muchas estrategias de comunicación rganizacional. La política, sin duda, no ha sido una excepción. Giansante (2015) ha glosado el potencial de Facebook como herramienta de comunicación política de manera tan exhaustiva a como lo ha hecho también Ricke (2014) con Youtube como caso de estudio, en su caso centrado específicamente en su impacto en la política norteamericana

No han sido las únicas redes sociales sobre las que se ha volcado el interés académico en torno a la nueva reconfiguración de las estrategias de comunicación política. Dado el objeto del caso de estudio propuesto, la exploración bibliográfica realizada para esta investigación se ha centrado, principalmente en Twitter.

\section{El impacto de Twitter en la comunicación}

\section{política. Una retrospectiva temática}

Lejos de ser únicamente una red social que aglutina enormes flujos de opinión, Twitter se ha consolidado en los últimos tiempos como una herramienta indispensable para crear tendencia (Harvey, 2014), un resultado inescapable del torrente de mensajes producido por su audiencia millonaria de productores y receptores de micromensajes. La facilidad para la interacción y la percepción de la plataforma como una mediación casi invisible ha catapultado la popularidad de una red que ha acabado por introducirse también en círculos profesionales, con la política como uno de los más señalados. La aparente reducción, en términos técnicos cuanto menos, de la distancia entre gobernantes y gobernados, quiza uno de los más notables factores de la creciente desafección hacia la esfera política en el nuevo siglo, ha hecho de Twitter que parecía brinda las herramientas perfectas para que la comunicación política encontrase un nuevo medio para reencontrarse con su público objetivo y viceversa.

Buena prueba de ello es que la presencia de partidos y líderes políticos en Twitter es un hecho cuasi incuestionable en la planificación de las estrategias de comunicación política actual. Parece ya bastante extendida la convicción entre los políticos contemporáneos de que es necesario estar en estas plataformas para reducir esa brecha que les separa de los ciudadanos y conseguir, así, mostrar e implementar una cercanía que no solo les reporte réditos sino que, además, les permit hacer llegar sus mensajes de manera más inmediata y menos mediada —o mediada según los intereses de sus equipos de comunicación y no por medios ajenos- (Gainous \& Wagner, 2014).

En este nuevo entorno los políticos expresan una voluntad de aparecer en el nuevo entorn social como prueba de espontaneidad y cercanía en un intento por presentarse como miembros de la esfera pública con un inquebrantable deseo de debatir con sus conciudadanos. Twitter, sin duda, ofrece esa posibilidad desde un punto de vista técnico; aunque cabría preguntarse si es efectivo que el político realmente tenga voluntad o siquier tiempo para poder exprimir estas potencialidade de interacción
Desde el punto de vista de la compañía quedan, eso sí, pocas dudas de que siempre es conveniente presentar la herramienta como un elemento potencialmente dinamizador del debate público y, en última instancia, de la democracia misma

\section{Estudios previos sobre el impacto de}

\section{Twitter en la Comunicación Política}

Pese a todo, incluso teniendo en cuenta la consabida consolidación de Twitter como herramien ta aceptada y usada por políticos, militantes y ciudadanos en general, siguen vigentes varias cuestiones sobre la eficacia con la que los políticos emplean Twitter y el cambio real que su empleo está efectuando en la esfera pública. En otras palabras, no todos los autores concuerdan en señalar de manera unívoca un impacto positivo de la herramienta de microblogging en la revitalización del debate político. Autores como Bernal y Congosto (2014) reconocen el poten cial de inmediatez que abre posibilidades ante las que los medios tradicionales, ni siquiera en sus más recientes reformulaciones digitales, apenas pueden competir, aunque esto no tiene por qué conducir a un exceso de optimismo.

En 2011, Parmelee y Bichard publicaron un estudio seminal en el que se subrayaba la importancia de Twitter en la explicación de los mecanismos de las campañas electorales modernas, algo que también ha llamado la atención de Héras-Pedrosa et al. (2019). No sería sensato obviar, además, el potencial democratizador que ofrece una herramienta que parece abrir las puertas a más actores en el proceso de definición de agenda, como bien señaló Shirky (2011) aquel mismo año. Resulta también lógico colegir que los propios políticos puedan estar usando Twitter como mecanismo de interacción, ya sea ésta directa o indirecta; en otras palabras, ya sea 
entrando en debates dialécticos con sus gobernados o siguiendo con atención el termómetro de opinión que constituyen los trending topics o los asuntos que, sin serlo, parecen ocupar a quienes interactúan con sus cuentas; extremo éste sobre el que también han llamado la atención Tumasjan et al. (2010)

El caso de la campaña electoral y posterior presidencia de Barack Obama es, sin duda, paradigmático en el estudio académico del influjo de Twitter en las estrategias de comunicación política. Al ya suficientemente glosado papel que la red de microblogging desempeñó en la campaña electoral de Obama en 2008 (cf. Borges, 2009 y Hendricks y Denton, 2010), su ex asesor Daniel Pfeiffer ha confirmado recientemente que Twitter fue una herramienta de importancia crítica en el traslado de los puntos de vista del Presidente a la ciudadanía (Pfeiffer, 2018)

Sin embargo, poco después podían leerse también algunas voces como las de Michailidou y Trenz (2010) o Vergeer, Hermans y Cunha (2012) que invitaban a una mayor precaución a la hora de asignar una valoración positiva al impacto de estas nuevas tecnologías en la esfera política. Los estudios a ambos polos de esta interpretación del impacto de Twitter sobre la esfera política comenzaron a proliferar a partir de esta década, con casos de estudio representativos como los de Holotescu et al. (2009) en Rumanía, Larsson yoe $(2011,2013)$ en Suecia y Dinamarca, Mambrey y Dörr (2011) en Alemania o Vergeer y Hermans (2013) en Holanda. Es también destacable el estudio de Lilleker y Jackson (2013), un análisis comparativo sobre el uso de Twitter en Reino Unido, Francia y Estados Unidos; por cuanto la investigación que proponemos triangulará, igualmente, en la comparación sobre los discursos en Twitter de tres líderes políticos en el contexto actualizado de nuestros días: el presi- dente de los Estados Unidos, Donald Trump, la primera ministra de Reino Unido, Theresa May, y el presidente del Gobierno de España, Pedro Sánchez. El interés, cuanto menos, del primero de ellos, ha suscitado interesantes reflexiones al respecto de la naturaleza de su discurso como las de Ahmadian et al. (2017) o Ashcroft (2016)

El caso español. El comienzo de la década de 2010 también fue un claro punto de partida en entorno académico español para el estudio de Twitter en la esfera política. Uno de los primeros puntos de inflexión fueron las Elecciones Generales de 2011, que Cebrián \& Vázquez (2013) estudiaron centrándose en las cuentas del líder del Partido Popular, Mariano Rajoy, y el candidato del Partido Socialista, Alfredo Pérez Rubalcaba. Su análisis concluía que las acciones de comunicación implementadas por los dos políticos a ravés de Twitter no se veían acompañadas por un incremento de la participación ciudadana. En una línea similar, y sobre la base del estudio de los mismos comicios, Zurutuza (2012) reafirma no solo esta ausencia de debate real sino una alta emejanza del empleo de la nueva plataforma con los usos tradicionales de la comunicación política, más centrada en la difusión de propuestas ideológicas, pero sin intención clara de crear o estimular un debate con quien antes resultaba técnicamente imposible hacerlo.

Las Elecciones Autonómicas de 2012 en Caaluña, Galicia, Andalucía y País Vasco llamó también la atención de los académicos españoes. Cebrián et al. (2013) y Deltell et al. (2013) arrojaron interesantes conclusiones en relación con los comicios en estas dos últimas comunidades autónomas. Su estudio de los mensajes de partidos y líderes políticos conducían a la misma conclusión: no había intención manifiesta de comenzar un debate público con un mínimo de originalidad y relevancia. Además, el diálogo en- tre gobernantes y gobernados era poco efectivo, por no decir inexistente.

Las Elecciones Europeas de 2014 marcaron el comienzo de un cambio político que se plasmó en el reparto electoral, con la entrada de partidos de cuño reciente. Esto llevó a cuestionars si el uso de las nuevas tecnologías y su empaste en las estrategias de comunicación política se encontraba entre los factores que habían llevado a este primer paso del quebrantamiento del bipartidismo. En su estudio de estos comicios, Gelado y Bonete (2017) sugerían que la presencia en Twitter de los políticos que concurrieron las elecciones no implicaba necesariamente que estos supieran cómo aprovechar las potencialidades de la herramienta ni que deseasen entrar en un debate efectivo con los ciudadanos. Siguiendo en la línea de lo demostrado por Zurutuza, Ge lado y Bonete (2017, p. 203) concluyeron también para estos comicios que «Twitter constituyó durante la campaña y en los días posteriores a la cita electoral un escaparate de información política y declaraciones destinado a cumplir con unos objetivos de comunicación más próximos al paradigma de los medios tradicionales de masa que a una verdadera voluntad de acercamiento los votantes y una patente interacción con ellos» Del mismo modo, se apuntaba también una línea de investigación que podía ser interesante: la simplificación del mensaje que estaba potenciando Twitter como plataforma de comunicación polí tica, el consiguiente vaciado de relevancia de los debates sugeridos, y la no excesiva diferenciación del tono de los discursos entre los partidos consolidados y los más emergentes.

\section{Metodología}

. presente estudio aspira a actualizar el estado de la cuestión del impacto de Twitter en el espacio público a través del análisis de los discursos de tres líderes políticos: el presidente de los Estados Unidos, Donald Trump, la primera ministra de Reino Unido, Theresa May, y el presidente de Gobierno de España, Pedro Sánchez.

\section{Diseño de la investigación}

Una vez elegidas las tres cuentas de los políticos anteriormente mencionados, seleccionados por la relevancia del impacto de su discurso a escala nacional e internacional, nos encontramos con la necesidad de acotar diversos aspectos de nuestr investigación. En primer lugar, el marco temporal, que por la aspiración de esta investigación a actualizar el debate sobre el impacto de Twitter en la esfera política debía arrojar una muestra, por un lado, lo suficientemente amplia como para que sus resultados fueran significativos y, por el otro, lo suficientemente reciente.

Después de realizar un primer estudio exploratorio de los tweets recopilados a partir de los que estaban disponibles en la API en el momento de realizar la investigación y con la idea de mantener una muestra que no bajase de las dos mil unidades textuales analizadas, se determinó estudiar las publicaciones emitidas desde las cuentas de presidente de los Estados Unidos, Donald Trump (@realDonaldTrump), la primera ministra de Reino Unido, Theresa May (@theresa_may) y e presidente del Gobierno de España, Pedro Sánchez (@sanchezcastejon) entre los meses de septiembre y diciembre de 2018. El universo final, coincidente con la muestra analizada, arrojaba una producción de 2.251 unidades textuales analizadas: 1.319 de la cuenta de Twitter de Donald Trump, 310 para la cuenta de Theresa May y 622 en la cuenta de Sánchez.

Para recopilar los tweets de las cuentas anteriormente mencionadas hemos usado el software Nvivo 11 que, a través de su add-on Ncapture permite recopilar los tweets almacenados en AP 
de manera sistemática y ofrece, además, la posibilidad de recuperar no solamente los tweets emitidos por esas cuentas sino también los retweets, lo cual resultaba interesante para medir las dimensiones del debate suscitado a través de esta vía indirecta. La recopilación sistemática de este corpus de análisis nos ha permitido afrontar la etapa de análisis tanto desde el punto de visto cualitativo inmanente a las disciplinas de estudio de la comunicación social como desde un punto de vista cuantitativo que confirmase los hallazgos obtenidos por la otra vía. De este modo, nuestro trabajo de investigación triangula (Lewis-Beck et al., 2004) entre el análisis cuantitativo asistido por ordenador a través de la herramienta anteriormente mencionada, NVivo, y el posterior análisis cualitativo textual (Paltridge, 2012) que permitiera confirmar o rechazar las dos hipótesis de trabajo, que de manera provisional afirmaban que:

- Los políticos en Twitter dan prevalencia a las herramientas retóricas que a los temas de política real.

- Twitter perpetúa estrategias de comunicación política más basadas en la promoción personal y de discursos afines que en la interacción real con los gobernados.

Para el caso de estudio propuesto las hipótesis previamente formuladas se testaron mediante el análisis de las tres cuentas de los líderes de Estados Unidos, Reino Unido y España durante el último cuatrimestre de 2018, o lo que es lo mismo, el principio del curso político 2018-19.

Una vez determinada la muestra y la herramienta tanto para el rescate como para el análisis de los tweets, se especificaron las categorías de análisis. Para el análisis de los datos se empleó un doble enfoque: por un lado el análisis cuantitativo realizado a través de las herramientas de medición de frecuencias de palabras de Nvivo y, por otro, un análisis cualitativo por el que se trascendía la mera medición cuantitativa de palabras para tratar de encontrar patrones discursivos en los micromensajes publicados por los candidatos sometidos a análisis. Para apoyar la visualización cuantitativa de los ejes discursivos predominantes en los mensajes de los políticos sometidos a estudio se recurrió a la herramienta de árboles conceptuales de Nvivo, vinculada a la búsqueda de frecuencias de palabras.

\section{Resultados}

El recuento de resultados de la muestra de tweets analizados arroja datos interesantes para cada uno de los candidatos. En e caso de Donald Trump, como se puede ver en la figura 1, se aprecia una corroboración cuantitativa de ejes discursivos no solo conocidos - la audiencia ya ha recibido a través de otros soportes desde el inicio de su carrera electoral- sino que desde una perspectiva cualitativa permite desnudar una carga dialéctica orientada mucho más hacia la retórica («great», «now», «people») que hacia temas políticos de gran calado como la economía o la educación. Esto redunda en dos fenómenos: por un lado, la consonancia, más que particularidad, de Twitter dentro de la estrategia de comunicación política de Trump: el presidente de los Estados Unidos utiliza la red de microblogging generalmente para mplificar su discurso o incluso para iniciarlo, no para ofrecer variantes adaptadas a las peculiaridades del nuevo medio, ni mucho menos para iniciar un diálogo con sus conciudadanos. La prevalencia de un discurso centrado en formulaciones en primera persona y la ausencia prácticamente absoluta de respuestas a otros tweets o e conversaciones en los más de mil tweets analizados del presidente estadounidense certifican este extremo. (Figura 1).

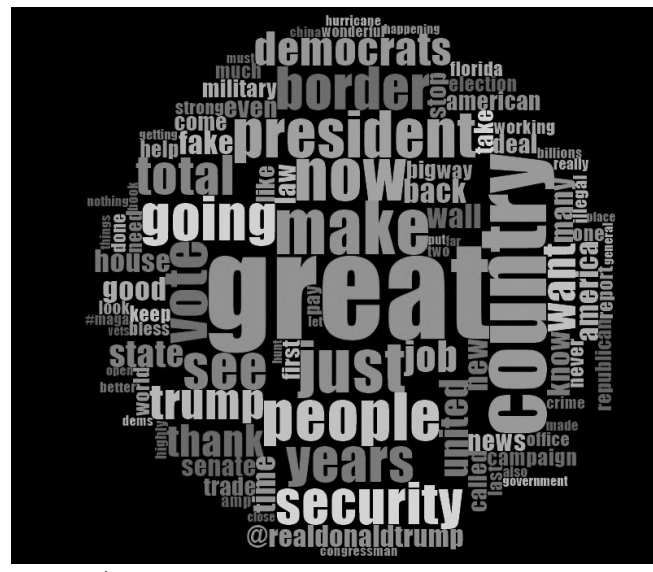

periodo sometido a estudio.

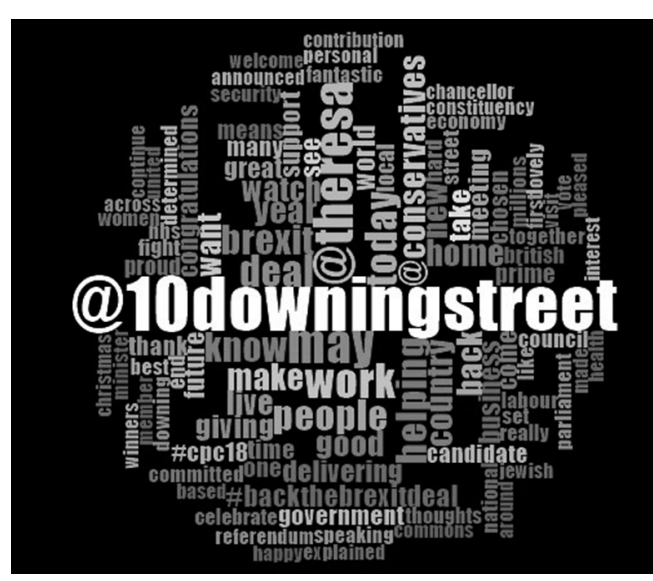

riodo sometido a estudio

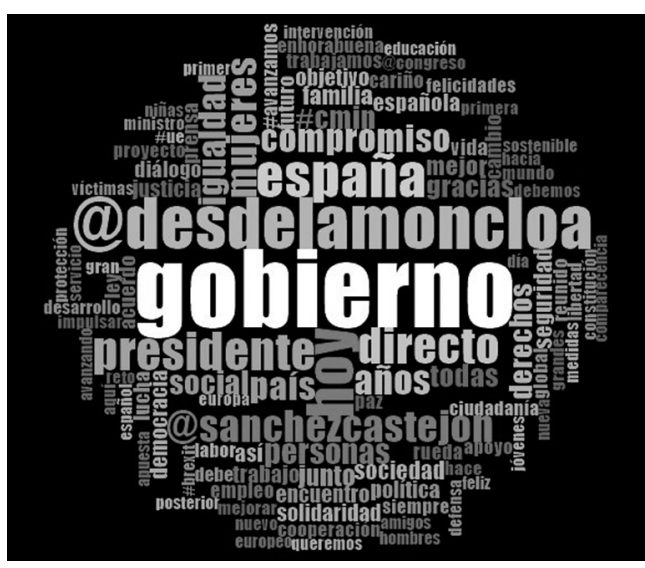
Figura 3. Árbol conceptual del discurso de Pedro Sánchez durante
periodo sometido a estudio.
El caso de la primera ministra del Reino Unido, Theresa May, corrobora esta tendencia al empleo de Twitter como escaparate político más que como voluntad de establecer un contacto efectivo con sus gobernados, como demuestra el hecho de que las menciones a la cuenta corporativa de Downing Street y a la suya propia sean dos de los ejes más prominentes de su discurso; algo que se puede observar en la figura 2. Twitter, en cualquier caso, acaba resultando para la máxima mandataria británica un lugar en el que estar y promocionarse mucho mas una herramienta con la que mejorar la calidad y la cantidad del debate público, como demuestra el hecho de las muy numerosas menciones a su propio partido, los conservadores o a la cancillería. En términos cualitativos, además de que el discurso de May orbite aún más en torno a sus cuentas de confianza, sí se observa una similar distribución de su discurso en torno a los mismos ejes discursivos que Trump: fundamentalmente retóricos y aislados de grandes temas políticos. (Figura 2)

Con el doble de tweets que Theresa May, pero aún a una misma distancia relativa de la profusa presencia tuitera de Donald Trump, el por entonces recién investido Presidente del Gobierno de España, Pedro Sánchez, tampoco emplea Twitter para entablar ningún diálogo real con actores de la esfera pública nacional. En la figura 3 se observa cómo las dos únicas cuentas que aparecen mencionadas en el árbol de frecuencia de palabras elaborado a partir de sus tweets son la suya propia y la institucional del Gobierno de España. Además el segundo valor más recurrente en ese gráfico después de las alusiones del presidente a la palabra «gobierno». Todos estos trazos vuelven a señalar en la dirección ya debatida para las dos cuentas mencionadas anteriormente: Pedro Sánchez emplea Twitter, como los otros dos políticos sometidos a análisis en esta investigación, como 
escaparate de sus políticas, de sus mensajes, y de sí mismo; no como herramienta para establecer un diálogo efectivo. Como May y Trump, entre las palabras más frecuentes de Sánchez no se encuentran grandes temas políticos, sino, esencialmente, mecanismos retóricos. (Figura 3)

\section{Conclusiones}

Una vez sometidas a estudio las tres cuentas analizadas, las conclusiones reafirman las hipótesis planteadas, según la cual es evidente que los politicos sometidos a estudio han incorporado Twitter como una herramienta más dentro de sus estrategias de comunicación política, si bien la emplean de un modo tan próximo al visto en los canales de comunicación tradicionales que cuesta encontrar factor diferencial que aporta Twitter dentro de sus estrategias. En otras palabras, los políticos analizados utilizan la red de microblogging como extensión de las tradicionales estrategias de repetición de mensajes clave y/o de información para su electorado potencial de cuanto hace dice el político. Las cuentas de Twitter analizadas durante el último cuatrimestre de 2018 son, en fin, mucho más un escaparate individual del político que un intento de apertura por parte de éste para involucrarse en un debate real con sus gobernados, como demuestra el hecho de que entre sus palabras más citadas solo aparecen como cuentas frecuentes las suyas propias o las de sus partidos y afines.

Es evidente, observando cuantitativamente la producción de mensajes en la red de microblogging, que este canal está incorporado a las estrategias de comunicación de los políticos estudiados y, como sabemos por estudios anteriores reseñados en nuestra revisión bibliográfica, también de los partidos. Sin embargo, resulta llamativa la escasa voluntad de interacción por parte de los políticos analizados, visible por un lado en el tipo de cuen- tas recurrentes que mencionan (con frecuencia, endogámicas: o bien las suyas propias o las de sus partidos o grupos de influencia afines) y, por el otro, en la observación del escaso número de respuestas a usuarios aleatorios, lo cual alimenta en sí el cuestionamiento de este pensado aminoramiento de la distancia entre los gobernantes y sus gobernados.

Del análisis se desprende también que tanto el tipo de temas como los enfoques abordados en sus cuentas de Twitter descubren a unos políticos que utilizan el nuevo canal de un modo muy similar al que utilizaban canales unidireccionales anteriormente. El objetivo de la mayor parte de las comunicaciones se limita a dar cuenta de dónde y qué se encuentra haciendo el político o, simplemente, a reproducir de una manera escasamente profunda las visiones políticas que tiene; o que, con frecuencia, evoca a los eslóganes de plataformas con menos opciones de interacción que las ofrecidas por aquellas emergidas al albur de la Web 2.0.

Del mismo modo, se puede concluir del nálisis de los tres políticos estudiados que sus discursos se articulan mucho más sobre ejes retóricos consonantes con sus declaraciones en medios tradicionales que sobre temas de política real que pueda estimular un verdadero debate entre gobernantes y gobernados. Sí se explota en las cuentas analizadas, sobre todo en el caso de Donald Trump y Pedro Sánchez, el factor de instantaneidad para dar una impresión de cercanía que sí permite una herramienta como Twitter. Sin embargo, es quizá también el modo más sencillo de esconder que la rapidez del formato no aporta una gran variedad sobre el método de comunicación unidireccional que históricamente ha prevalecido en la comunicación política y que, de momento, Twitter tampoco parece haber sido capaz de cambiar; al menos no radicalmente.
Por todo ello, nuestro estudio concluye que no hay datos para afirmar que Twitter ha tenido un impacto especialmente significativo en la reactivación de la esfera pública a través de la política o de acercamiento de sus dos actores principales, los políticos y sus representados. Con todo, la naturaleza

\section{Bibliografía}

Ahmadian, S., Azarshahi, S. \& Paulhus, D. L. (2017). Explaining Donald Trump via communication style: Grandiosity informality and dynamism. Personality and Individual Differences, n. 107, 49-53. doi:10.1016/j.paid.2016.11.018 Ashcroft, A. (2016). Donald Trump: Narcissist, Psychopath or Representative of the People? Psychotherapy and Politics International, 14(3), 217-222. doi:10.1002/ppi.1395

Bernal, A.I. y Congosto, M. (2014). Campaña electoral de las elecciones europeas: medios de comunicación vs. viralidad de la Red, Disponible en http://www.alice-comunicacionpolitica. com/abrir-ponencia $p h p 2 f=508-F 5422 a 58 d 5081411$ 556749 -ponencia-1.pd

Borges, B. (2009) Marketing 20: Bridging the Gap Between Seller and Buyer Through Social Media Marketing. Tucson, Arizona: Wheatmark

Cebrián, E. y Vázquez, T. (2013). @marianorajoy VS @conRubalcaba: La campaña electoral de 2011 en las cuentas oficiales de Twitter de los candidatos del PP y del PSOE. En Miradas a las pantallas en el bolsillo (pp. 93-108). Madrid: Universidad Camilo José Cela.

Cebrián, E., Vázquez, T. y Olabarrieta, A. (2013). ¿Participación y democracia en los medios sociales?: El caso de Twitter en las elecciones vascas de 2012, adComunica, 6, 39-63.

Deltell, L., Claes, M. y Osteso, J.M. (2013). Predicción de tendencia politica por Twitter: Elecciones Andaluzas 2012 Ámbitos, 22.

Fuchs, C. (2013). Social Media: A Critical Introduction London: Sage.

Gainous, J. \& Wagner, K.M. (2014). Tweeting to Power: The Social Media Revolution in American Politics. Oxford: Oxford University Press. cambiante del fenómeno comunicativo en general y de las redes sociales en particular invitan a una revisión constante de los resultados, por lo que es necesario realizar futuras investigaciones que sigan arrojando luz y actualicen el impacto de las nuevas formas de comunicación en las estrategias políticas.
Gelado, R. y Bonete, F. (2017). La competencia digital de los politicos españoles en Twitter. El caso de las elecciones europeas de 2014, edmetic, 6(2), E-ISSN: 2254-0059; pp. 185-209. doi: https://doi.org/10.21071/edmetic.v6i2.6929

Giansante, G. (2015). Online Political Communication: How to Use the Web to Build Consensus and Boost Participation. New York: Springer

Harvey, K. (2014). Encyclopedia of Social Media and Politics. Los Angeles: Sage.

Hendricks, J. H (2010) Communicator-in-Chief: How Barach Obama Used New Media Technology to Win the White House. Nueva York: Lexington Books.

Heras-Pedrosa, C., Jambrino-Maldonado, C., Iglesias-Sánchez, P. \& Lugo-Ocando, J. (2019). The USA presidential campaign from an European viewpoint. The public relations strategic and the power of images. Revista Norteamenica.

Holotescu C., Gutu, D., Grosseck, C., y Bran, R. (2009) Micro-blogging meets politics. The influence of communication in 140 characters on Romanian presidential election in 2009 Romanian Journal of Communication and Public Relations, 13(1), 37-47.

Holmes, J. W. \& McNeal, R. S. (2016). Social Media, Participation, and Attitudes: Does Social Media Drive Polarization? En Tomaž Deželan (Ed.). (R)evolutionizing Political Communication through Social Media. Hershey (PA): IGI Global, 1-21. Richey, S. (2013). The Social Basis of the Rational Citizen: How Political Communication in Social Networks Improves Civic Competence. Lanham: Lexintong Books.

Larsson, A. y Moe H. (2011). Studying political microblogging. Twitter users in the 2010 Swedish election campaign, New Media and Society, 13(7), 1.180-1.197. 
Larsson, A. y Moe H. (2013). Representation or participaion? Twitter use during the 2011 Danish election campaign, Javnost-The Public, 20(1), 71-88

Lewis-Beck, M., Bryman, A. E., Futing Liao, T. (2004). The SAGE Encyclopedia of Social Science Research Methods. Londres: Sage.

Lilleker, D. y Jackson, N. (2013). Political campaigning, elections and the Internet: Comparing the US, UK, France and Germany. Londres: Routledge

Mambrey, P. y Dörr, R. (2011). Local government and socia networking technologies in Germany: The example of Twitter. Conference for E- Democracy and Open Governmen (pp. 249-260).

Michailidou, A. y Trenz, H.J. (2010). Mediati(zi)ng EU politics: Online news coverage of the 2009 European Parliamentary elections, Communications, 35(3), 327-346.

Paltridge, B. (2012). Discourse Analysis: An Introduction Nueva York: Bloomsbury.

Parmelee, J. H., Bichard, S. L. (2011). Politics and the twitter revolution. How tweets influence the relationship between politcal leaders and the public. Lexington books, Maryland.

Pfeiffer, D. (2018). Yes We (Still) Can: Politics in the age of Obama, Twitter and Trump. Biteback Publishing.

Ricke, L.D. (2014). The Impact of YouTube on U.S. Politic London: Lexington Books.

Semeitko, H. A. \& Scammell, M. (2012). The SAGE Handbook of Political Communication. London: Sage.
Shirky, C. (2011). The Political Power of Social Media. Technology, the Public Sphere, and Political Change, Foreig Affairs, 90(1).

Tumasjan, A., Sprenger, T.O. Sandner, P.G. y Welpe, I.M. (2010). Predicting Elections with Twitter: What 140 Characters Reveal about Political Sentiment. Proceedings of the Fourth In ternational AAAI Conference on Weblogs and Social Media. 178-185. Munich: Technische Universität München.

Vatikiotis, P. (2016). Social Media Activism: A Contested Field. En Tomaž Deželan (Ed.). (R)evolutionizing Political Communication through Social Media. Hershey (PA): IGI Global, 40-55.

Vergeer, M., Hermans, L. y Cunha, C. (2012). Web campaigning in the 2009 European Parliament elections: A ross-national comparative analysis, New Media \& Sociely, $15(1), 128-148$

Vergeer, M. y Hermans, L. (2013). Campaigning on Twitter: Microblogging and Online Social Networking as Campaign Tools in the 2010 General Elections in the Netherlands, Journal of Computer-Mediated Communication, 18 399-419.

Zurutuza, C. (2012, julio 6-7). ¿De qué habla Rubalcabe cuando tuitea? La campaña del candidato socialista en Twitter para las elecciones del 20N. Ponencia presentada para la mesa «Redes Sociales y Campañas Políticas: el uso de Twitter Facebook por los candidatos». I Congreso Internacional en Comunicación Política y Estrategias de Campaña, Madrid, España 